\title{
Perception of Patients about Cardiovascular Disease (CVD) and Effect of Communication by Physician and the Assisting Registered Nurse to Enhance Assessment of Risk and Bridge a Gap of Accurate Perception of their Risk of CVD
}

\author{
Article by Anette Marina Rodrigues \\ Nursing, Texila American University, India \\ E-mail: arodrigues@texilaconnect.com
}

\begin{abstract}
This paper validates the level of perception of patients about Cardiovascular Disease (CVD) and effect of communication by Physician and the assisting Registered Nurse to enhance risk assessment and bridge a gap of accurate perception of their risk of CVD.

Design and Procedure-A Non-experimental Descriptive study of patients visit to Out Patient Department for Physician Consultation. Non- Random selection of patients with complaints of chest pain visit from October 2016 to March 2017 over a period of 6 months. Assessment of Vital Parameters of 60 patients, Male-57 and Female 3, within age group of 30 to 40 years and ECG graphs readings were done for all. Information is collected from Health Information System Portal.

Result-Communication and providing positive thought on seriousness, to prevent CVD over the future years, among 60 patients with complaints of chest pain was a challenging aspect. $(n=15)$ were newly diagnosed with Hypertension, $(n=10)$ were follow up patients with Hypertension and Diabetes Mellitus), and $(n=25)$ were referred to Cardiologist with appointment fixed for further management and $(n=10)$ ECG was normal significant of gastric pain.

The Physician did initial assessment and significant symptoms on history of illness on all 60 patients. $(n=44)$ were advised on appropriate diet and reduction of weight by balanced diet and exercise. $(n=30)$ were advised on follow up among them $(n=25)$ had visited the physician as follow-up. $(n=10)$ Obese were referred to dietician with appointment fixed, and assisting nurse gave advice to $(n=50)$.

Conclusion-Patient's perception on CVD suggests $(n=50)$ were ignorant about risk of CVD and $(n=10)$ had known about risk of CVD. The trustful patient relation and communication by the treating Physician and health education by Registered Nurse has direct and positive effect on outcome of the research. Patients were enthusiastic to gain more knowledge on Modifiable risk factors. The attitude and behavior of patients were significant and positive with revisit and follow up visits.
\end{abstract}

Keywords: Perception, Cardiovascular Disease, Communication, Health Information System, Health education, follow up visit.

\section{Introduction}

\section{Purposes}

1) Identify patient's perception of cardiovascular risk.

2) Effective communication by treating Physician and Registered Nurse to bridge a gap.

3) Positive outcome of changing Modifiable Risk factors.

\section{Problem}

Cardiovascular Disease is the leading cause of death and disability worldwide. $80 \%$ of Acute Myocardial Infarction (Ref1) can be attributed to just five Modifiable Risk factors like Smoking, Dyslipidemia, Hypertension, Diabetes Mellitus and Obesity which are also implicated in the increased risk of Stroke. 
DOI: $10.21522 /$ TIJNR.2015.03.02.Art016

ISSN: $2520-3126$

\section{Existing solution}

Once the risk has been assessed treatment options are established. Statin Therapy, BP lowering medications, Antiplatelet Medications, Smoking Cessation and Diet and Exercise. Mass media and Mobile apps give lot of exercise schedule plans, diet modification and weight loss programs. But with one to one interaction with the General Practitioner or Physician will help the patient to clear the negative perceptions and change existing Modifiable Risk factors.

\section{Achievements}

Patient's perception of Cardiovascular risk shows their attitude and behavior is changed with the details in the figures of patients revisit to Physician and with follow up cases for further investigation, medications, dietary modifications and advanced cardiology visit.

Effective communication by treating Physician and Registered Nurse to bridge a gap of positive outcome of changing Modifiable Risk factors. This is evident in initial visit and follow up visit.

\section{Methods}

\section{Description of setting}

Observations of patients visit to General practitioner and Physician.

Demographic Data/ History Collection.

Secondary data Collection of patient health record via Electronic health records.

High risk score calculator for 10 years.

\section{Population}

Total of 1200 patients of different age groups had visited the facility in six months for various specialists out of them 350 visited the Physician. Patients with symptoms of chest pain, sample size 60 patients (Male and Female) age group of 30 to 40 years had visited facility from October 2016 to March 2017 were the target.

\section{Variables}

\section{Exclusive criteria}

Patient with CVD, on treatment and surgery

\section{Inclusive criteria}

Age group 30-40 years

Symptoms of chest pain (Unknown Origin)

Known History of Hypertension and Diabetic Mellitus.

\section{Research approach}

The Physician has done detailed Physical Assessment and filled GHA form to identify the reason for symptoms like chest pain in 60 patient was taken on all patients. Blood Investigations like Serum Cholesterol, HDL Levels was done in few patients suspecting CVD. Patients with signs of palpitation, Sinus Tachycardia and Bradycardia of unknown and known origin were referred to Cardiologist. The Physician has explained about Modifiable Risk Factors like control Blood Pressure, Diabetes Mellitus, reduction and stopping of Cigarette smoking, overweight management and patients with increased BMI were given Dietician reference and advice for follow up. The assisting Registered Nurse has also advised on weight reduction, dietary control, exercise, intake of balanced diet and consume regular medications for Hypertension and Diabetes Mellitus as prescribed and follow up. 


\section{Research design}

All participants within age group of 30 to 40 years were the target out of 350 patients visit to Physician for various symptoms like Hypertension, Diabetic Mellitus, 60 patients with complaints of chest pain in 6 months were the target for the research. The knowledge, attitude and behavior related to risk of CVD is assessed during the triage and initial and follow up assessment by Registered Nurse and Physician. All the details are compiled and noted in Electronic Health Record.

Anthropometric and Demographics study including Age, Nationality, BMI, education level was identified on the participants by Receptionist with OPD registration, RN with Anthropometric measurements and vital parameters recording

Observation method was used to assess the type, location and severity of chest pain; non-biased random selection with assessment of their knowledge, new diagnosis management, follow up treatment, referral to Cardiology and Dietician was done by treating doctor.

Cardiovascular risk score is calculated to identify risk in next 10 years which is not a part of Electronic Health Record.

\section{Variables with measuring devices}

Continuous Variable, Bio physiological Measurement like Blood Pressure, Pulse, Blood Sugar, Serum Cholesterol Levels and HDL Levels were compared with initial assessment and follow up depending on the symptoms and ECG findings.

\section{Setting of the study}

The study is done in Out Patient Department of NMC Medical Center with 5 General Practitioners, 1 Physician and 10 Specialists. The patients were directed to Physician with internal referral from GP Doctor according to Insurance Coverage and direct reference to Physician (Internal Medicine) with Insurance coverage and cash bases too.

\section{Result}

1) Patient's perception of cardiovascular risk was assessed before the visit to physician, during triage and after visit to physician, while providing health education to patient and family.

2) Effective communication by treating Physician and Registered Nurse to bridge a gap was done during the triage, consultation and after care of patient.

3) Positive outcome of changing Modifiable Risk factors is with graphic representation of patient revisit to the physician and dietician. Cardiovascular risk score is calculated to identify risk in next 10 years

\section{Discussion}

Patients perception on CVD suggests $(n=50)$ were ignorant about their health status initially and knowledge about risk of CVD and $(n=10)$ had known about risk of CVD. The trustful patient relation and communication by the treating Physician and Registered Nurse has direct and positive effect on outcome of the research Patients were enthusiastic to gain more knowledge on Modifiable risk factors. The attitude and behavior of patients were significant and positive with follow up visits and regular intake of medications. Similar study was done by Ruth Webster; Perceptions of risk: understanding cardiovascular disease (Ref 1) with need to educate the patient.

Cardiovascular risk score is calculated to identify risk in next 10 years Health. Out of 33 patients with blood values of Cholesterol and HDL, 21 patients have 0 to 10\% risk, 6 patients have 10 to $20 \%$ risk and 6 patients have 20 to $30 \%$ risk of CVD. (Ref 3 ). 
DOI: $10.21522 / \mathrm{TIJNR} .2015 .03 .02 . A r t 016$

ISSN: $2520-3126$

\section{Additional findings}

Routine General Health checkup must be advised to all persons in younger age groups of 25 to 45 . This will help to identify the initial risks and future risks of CVD.

The Health Information system (Electronic data recording) need to be advanced with High risk score calculator inbuilt to have wider and effective communication with the details of patient health outcomes instantaneously so that patient would have quick results and guidance to take appropriate steps to avoid risk of CVD.

\section{General take home message}

Each Individual is responsible for his/her own health. Balance of health and lifestyle modification need to be maintained in order to live without any disease. Physical and Mental wellbeing are both necessary to remain healthy. Regular General Checkup in younger age groups would definitely avoid future CVD Risk and Vascular Disorders like Hypertension and Endocrine Disorder like Diabetic Mellitus.

\section{Conclusion}

Patients perception on CVD suggests $(n=50)$ were ignorant about their health status initially and knowledge about risk of CVD and $(n=10)$ had known about risk of CVD. The trustful patient, physician communication by the treating Physician and Registered Nurse has direct and positive effect on outcome of the research Patients were enthusiastic to gain more knowledge on Modifiable risk factors. The attitude and behavior of patients were significant and positive with follow up visits. Cardiovascular risk score is calculated to identify risk in next 10 years Health Risk for CVD which is very significant.

\section{Figures and tables}

Table 1. Comparison-before/after scores

\begin{tabular}{|l|l|l|}
\hline Personal Data & Risk factors & Value in percentage \\
\hline Age Group & $30-40$ yrs. & 60 patients \\
\hline Sex & Male-(n=57) Female-(n=3) & \\
\hline Nationality & Indian-30, Pakistan-14 & \\
\cline { 2 - 3 } & $\begin{array}{l}\text { Bangladesh-9, Sudhanese- } \\
3\end{array}$ & \\
\cline { 2 - 3 } & Ethiopian-1, Comeron-1 & \\
\cline { 2 - 3 } & Jordan-1, Indonesian-1 & \\
\hline \multirow{5}{*}{ Weight } & $50-70 \mathrm{Kg}(\mathrm{n}=9)$ & $15 \%$ \\
\hline & $71-90 \mathrm{Kg}(\mathrm{n}=35)$ & $58 \%$ \\
\hline & $91-110 \mathrm{Kg}(\mathrm{n}=15)$ & $25 \%$ \\
\hline & $111-130 \mathrm{Kg}(\mathrm{n}=1)$ & $1.6 \%$ \\
\cline { 2 - 3 } & $140-160 \mathrm{Kg}(\mathrm{n}=1)$ & $1.6 \%$ \\
\hline \multirow{5}{*}{ BMI } & $20-24.9=(\mathrm{n}=18)$ & $30 \%$ \\
\hline & $25-29.9=(\mathrm{n}=20)$ & $33.3 \%$ \\
\hline & $30-40=(\mathrm{n}=22)$ & $37 \%$ \\
\hline
\end{tabular}




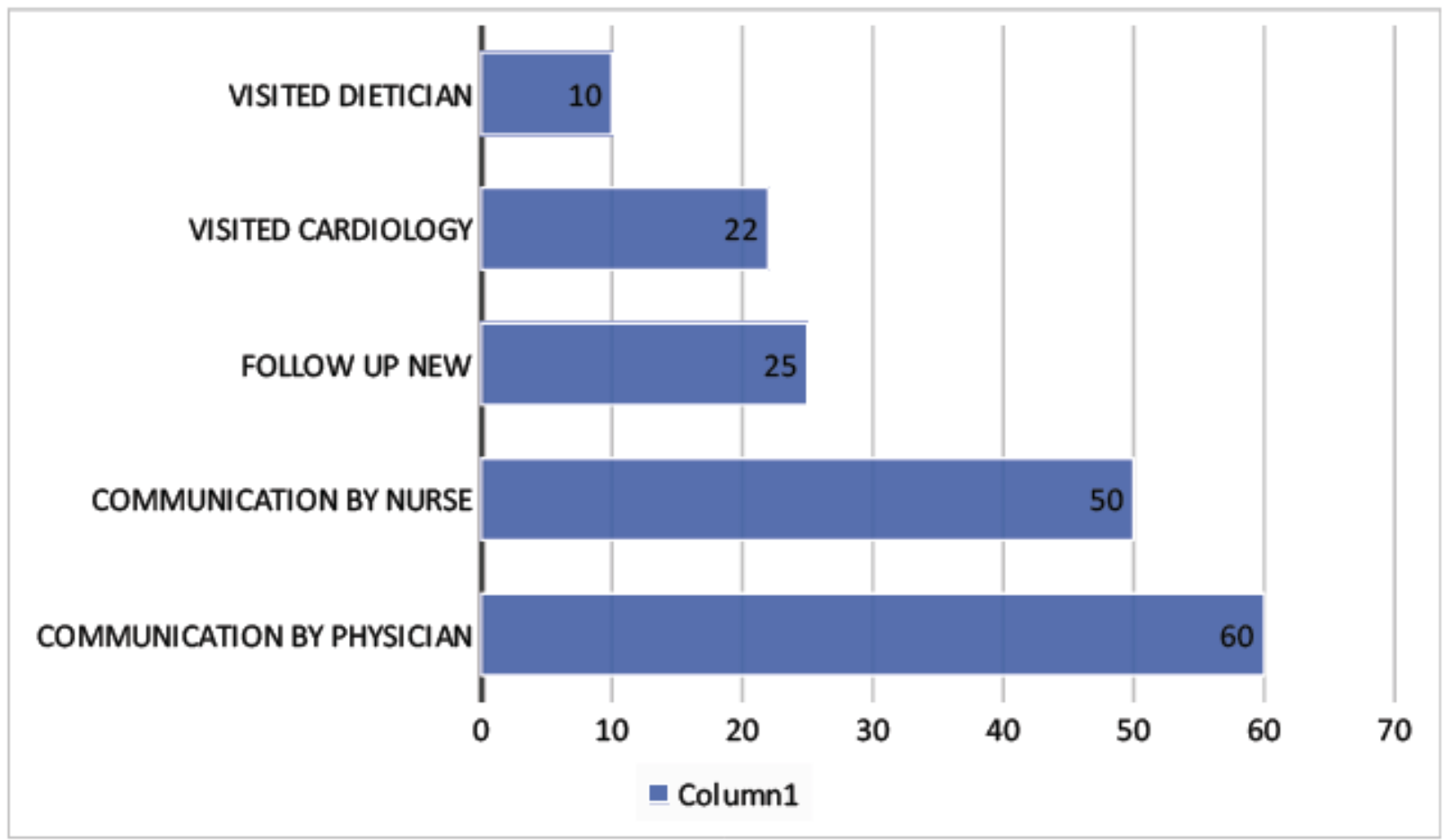

Figure 1. Physician assessment

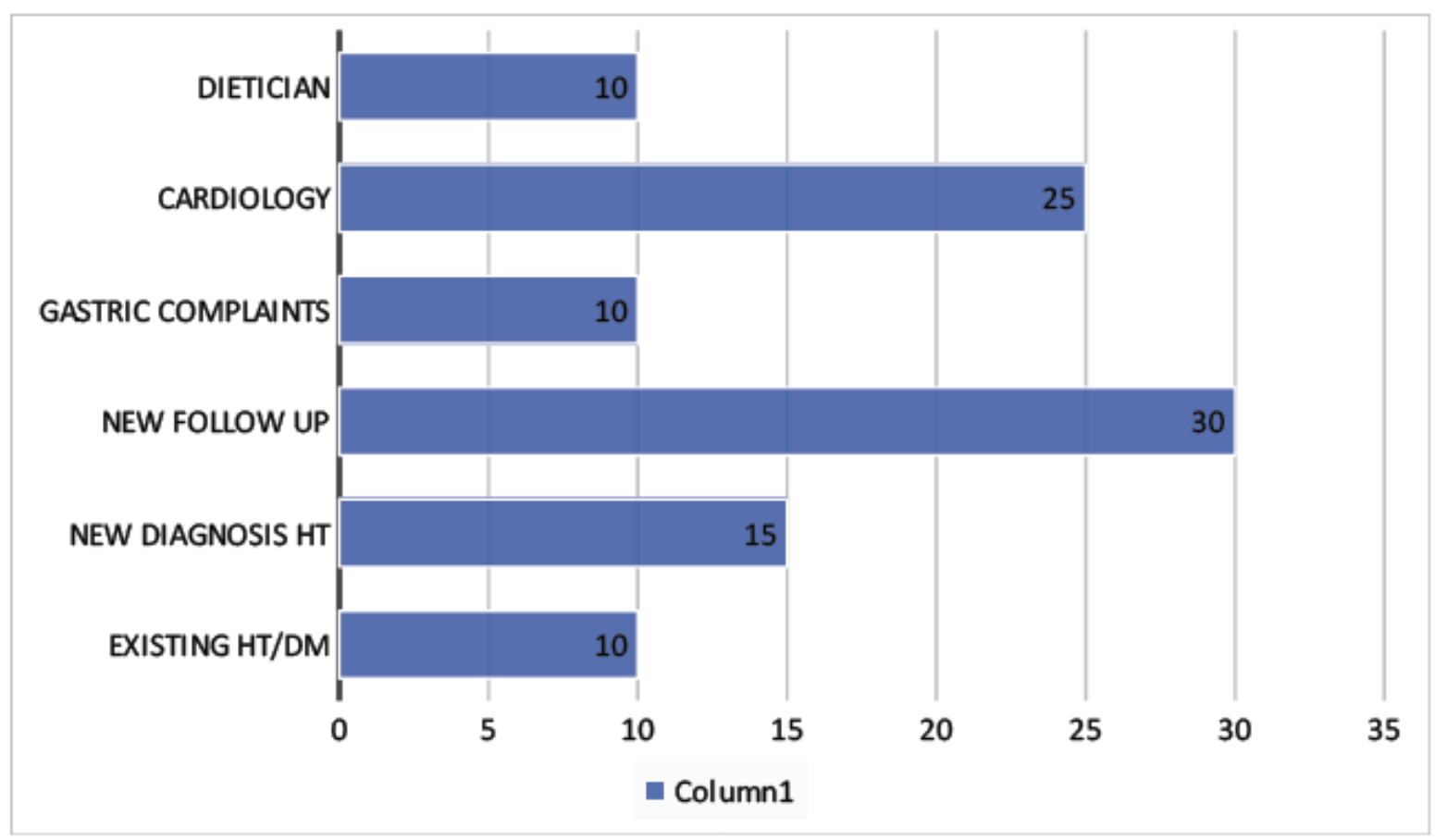

Figure 2. Follow up 
DOI: $10.21522 / \mathrm{TIJNR} .2015 .03 .02 . A r t 016$

ISSN: $2520-3126$

Calculator: Cardiovascula $\times$ Secure | https://www.uptodate.com/contents/calculator-cardiovascular-risk-assessment-10-year-men-patient-education
Calculator: Cardiovascular risk assessment (10-year, men: Patient education)
The content on the UpToDate website is not intended nor recommended as a substitute for medical advice, diagnosis, or treatment. Always seek the advice of your own physician or other qualified health care professional regarding any medical
questions or conditions. The use of this website is governed by the UDTODate Terms of Use $\Theta 2017$ UpToDate, Inc.

Calculator: Cardiovascular risk assessment (10-year, men: Patient education)

\begin{tabular}{|c|c|c|c|}
\hline \multicolumn{4}{|c|}{ Input: } \\
\hline Age & & $\mathrm{yr}$ & v \\
\hline Systolic blood pressure & & $\mathrm{mmHg}$ & v \\
\hline Total cholesterol & & $\mathrm{mg} / \mathrm{dL}$ & v \\
\hline HDL cholesterol & & $\mathrm{mg} / \mathrm{dL}$ & $\checkmark$ \\
\hline On blood pressure medication & No & & \\
\hline Cigarette smoker & No & & V \\
\hline Diabetes present & No & & , \\
\hline
\end{tabular}

Notes

- This calculator may be applied to men who have had no prior history of cardiovascular disease.

- A person with cardiovascular disease is someone who has (now or in the past) blocked coronary arteries, a heart attack, a stroke or blocked carotid arteries, peripheral arterial disease (blocked arteries to the legs), or heart failure.

- Systolic blood pressure is the top number (eg, 120 if blood pressure is $120 / 80$ )

Cardiovascular risk calculator (Part 1) (Ref 3)

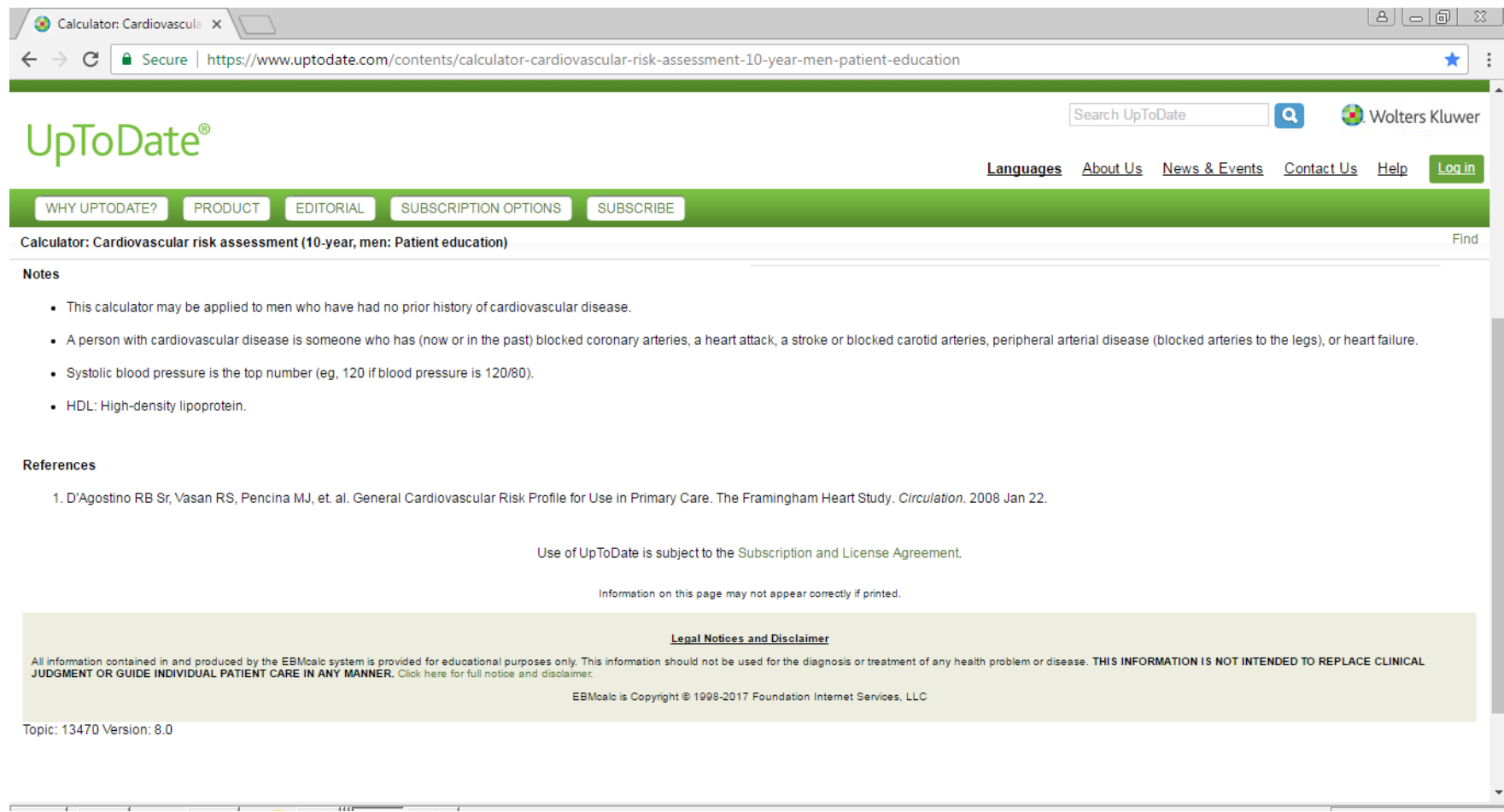

Cardiovascular risk calculator (Part2) 


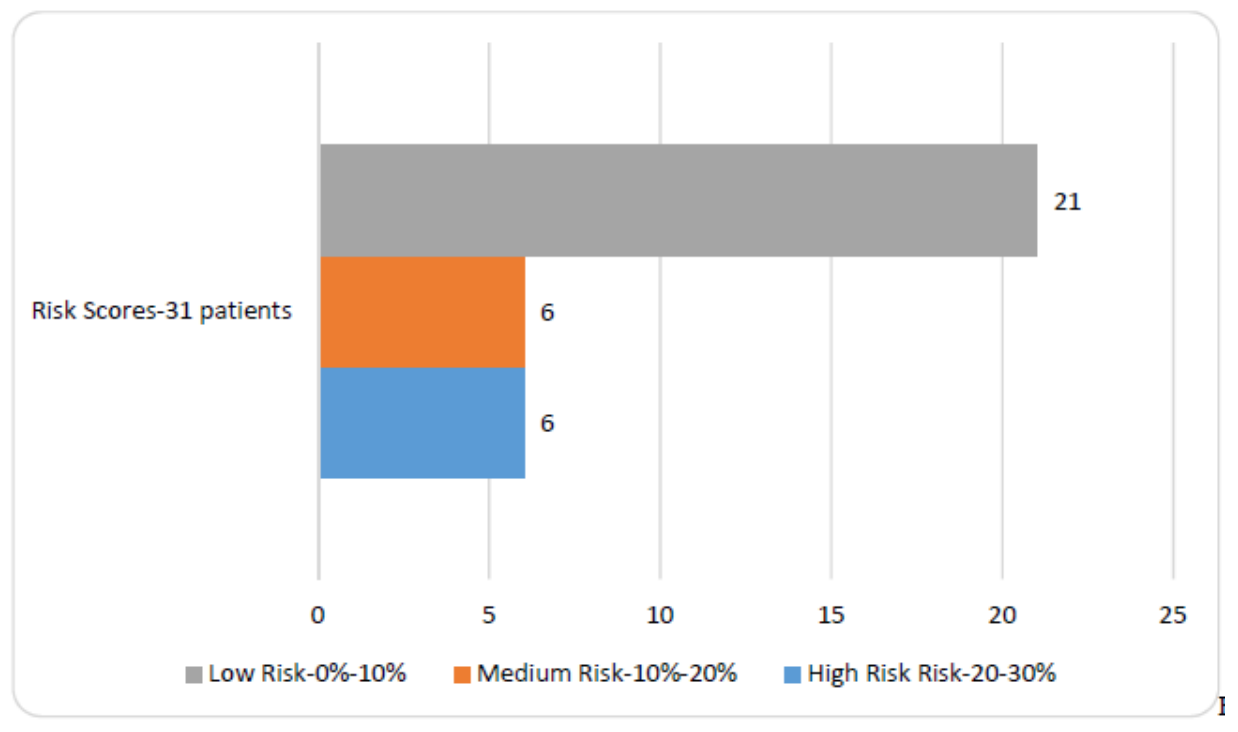

\section{Acknowledgement}

High risk score calculator results

I thank my facility, NMC Medical Centre, Alwadi for giving me an opportunity to do the Primary Research.

I thank the Physician and staff nurses, Registered Nurses for assisting me in doing the research and providing health education to the patients.

I thank the patients who are part of this project.

\section{References}

[1].Peterson JA; One theoretical framework for cardiovascular disease prevention in women; Journal of Cardiovascular Nursing. 2012 Jul-Aug; 27(4):295-302. doi: 10.1097/JCN.0b013e31822072fc.

[2].Ruth Webster; Perceptions of risk: understanding cardiovascular disease; Dove Press Journal; 2010 Webster and Heely, publisher and licensee Dove Medical Press Ltd; Published 4 September. https://www.ncbi.nlm.nih.gov/pmc/articles/PMC3270917/.

[3].https://www.uptodate.com/contents/calculator-cardio vascular-risk-assessment-10-year-men-patient-education. 